\title{
Perinatal outcomes of neonates born from different endometrial preparation protocols after frozen embryo transfer: a retrospective cohort study
}

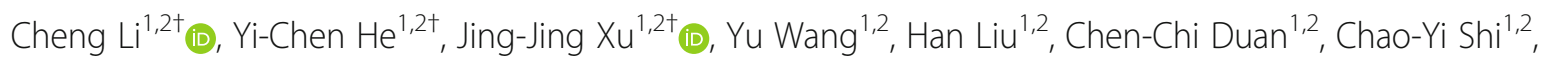
Lei Chen ${ }^{1,2}$, Jie Wang ${ }^{1,2}$, Jian-Zhong Sheng ${ }^{3}$, He-Feng Huang ${ }^{1,2,4^{*}}$ (i) and Yan-Ting Wu ${ }^{1,2,4^{*}}$ (D)

\begin{abstract}
Background: Previous studies have focused on pregnancy outcomes after frozen embryo transfer (FET) performed using different endometrial preparation protocols. Few studies have evaluated the effect of endometrial preparation on pregnancy-related complications. This study was designed to explore the association between different endometrial preparation protocols and adverse obstetric and perinatal complications after FET.

Methods: We retrospectively included all FET cycles $(n=12,950)$ in our hospital between 2010 and 2017, and categorized them into three groups, natural cycles (NC), hormone replacement therapy (HRT) and ovarian stimulation (OS) protocols. Pregnancy-related complications and subsequent neonatal outcomes were compared among groups.

Results: Among all 12,950 FET cycles, the live birth rate was slightly lower for HRT cycles than for NC (HRT vs. NC: $28.15 \%$ vs. $31.16 \%, p<0.001)$. The pregnancy loss rate was significantly higher in OS or HRT cycles than in NC (HRT vs. NC: $17.14 \%$ vs. $10.89 \%, p<0.001$; OS vs. NC: $16.44 \%$ vs. $10.89 \%, p=0.001$ ). Among 3864 women with live birth, preparing the endometrium using OS or HRT protocols increased the risk of preeclampsia, and intrahepatic cholestasis of pregnancy (ICP) in both singleton and multiple deliveries. Additionally, OS and HRT protocols increased the risk of low birth weight (LBW) and small for gestational age (SGA) in both singletons and multiples after FET.
\end{abstract}

Conclusion: Compared with HRT or OS protocols, preparing the endometrium with NC was associated with the decreased risk of pregnancy-related complications, as well as the decreased risk of LBW and SGA after FET.

Keywords: Frozen-thawed embryo transfer, Endometrium preparation, Gestational hypertensive disorder, Intrahepatic cholestasis of pregnancy, Small for gestational age

\footnotetext{
* Correspondence: huanghefg@sjtu.edu.cn; yanting_wu@163.com

${ }^{\dagger}$ Cheng Li, Yi-Chen He and Jing-Jing Xu contributed equally to this work. 'International Peace Maternity and Child Health Hospital, School of Medicine,

Shanghai Jiao Tong University, No.910, Hengshan Rd., Shanghai 200030,

China

Full list of author information is available at the end of the article
}

(c) The Author(s). 2021 Open Access This article is licensed under a Creative Commons Attribution 4.0 International License, which permits use, sharing, adaptation, distribution and reproduction in any medium or format, as long as you give appropriate credit to the original author(s) and the source, provide a link to the Creative Commons licence, and indicate if changes were made. The images or other third party material in this article are included in the article's Creative Commons licence, unless indicated otherwise in a credit line to the material. If material is not included in the article's Creative Commons licence and your intended use is not permitted by statutory regulation or exceeds the permitted use, you will need to obtain permission directly from the copyright holder. To view a copy of this licence, visit http://creativecommons.org/licenses/by/4.0/ The Creative Commons Public Domain Dedication waiver (http://creativecommons.org/publicdomain/zero/1.0/) applies to the data made available in this article, unless otherwise stated in a credit line to the data. 


\section{Background}

Frozen-thawed embryo transfer (FET) has been increasingly used in assisted reproductive technology (ART) to avoid the inferior effects of fresh embryo transfer, which include high E2 levels, and to surplus embryos after oocyte retrieval and in vitro fertilization (IVF) to improve cumulative pregnancy rates $[1,2]$. As laboratory techniques have improved, especially those related to the enhanced survival and implantation rates achieved by vitrification, the number of FET cycles performed has increased dramatically over the last decade [3].

The synchronization of embryo and endometrial development is regarded as a crucial factor in the success of FET. In contrast to the complicated controlled ovarian hyperstimulation protocols employed to stimulate follicular growth for IVF, it is much simpler to prepare the endometrium with the aim of improving its receptivity. Endometrial preparation protocols can be commonly divided into three methods: natural cycles (NC) using spontaneous ovulation, ovarian stimulation (OS) protocols performed with endogenous steroids, and hormone replacement therapy (HRT) protocols involving artificial preparation with exogenous steroids [4, 5]. Many cohorts have been studied and randomized controlled trials performed to evaluate the effects of these endometrial preparation protocols on pregnancy outcomes for FET; however, their findings remain controversial [5-8]. Although preparing the endometrium with exogenous hormones provides great advantages, such as minimizing monitoring and scheduling the timing of the procedure, the abnormal hormone levels observed during the window of implantation and the dysfunction of hormone response elements on endometrium induced by the HRT or OS protocols should not be ignored [9-11]. Our previous study has reported that the risk of adverse obstetric and neonatal outcomes after FET is increased by ovarian stimulationinduced superphysiological estradiol levels, potentially influencing the future health of offspring produced using this technique [12]. Compared to the pregnancy outcomes achieved after FET, few studies have explored the effect of endometrial preparation on pregnancy-related complications and subsequent neonatal outcomes.

Understanding the effects of different endometrial preparation protocols on pregnancy-related complications and perinatal outcomes can further optimize maternal and child health after FET. Therefore, this cohort study was designed to explore the associations between maternal exposure to different endometrial preparation protocols and perinatal outcomes, including pregnancy rates, adverse obstetric complications, and neonatal outcomes.

\section{Methods}

\section{Study design and participants}

We retrospectively recruited all infertile women undergoing FET at International Peace Maternity and Child
Health Hospital from May 2010 to September 2017. Women who underwent preimplantation genetic testing (PGT). All Chinese participants were categorized into three groups according to the protocols of endometrial preparation (nature cycles, OS protocols, or HRT protocols). Participants with live birth deliveries were included in the perinatal outcome analysis.

All procedures and follow-up performed in this study involving human participants were conducted in accordance with the ethical standards of the Institutional Review Board of the International Peace Maternity and Child Health Hospital, Shanghai, China (GKLW-2016-21). The reporting of this study conforms to the STROBE statement.

\section{ART procedures}

The process of ART was conducted as we previously described [12]. Briefly, infertile women undergo controlled ovarian hyperstimulation $(\mathrm{COH})$, oocyte retrieval, and then insemination by either conventional in vitro fertilization (IVF) or intracytoplasmic sperm injection (ICSI). For women undergoing FET, embryos are cryopreserved via vitrification. Before embryo thawing, the endometrium was prepared by natural monitoring, HRT or an OS cycle.

Normally, NC was the favorable choice for women with regular menstruation. HRT or OS cycle was considered as preferred choice for women with irregular menstruation or history of anovulation. For women underwent NC, no medication was administrated during the follicular phase. For women underwent HRT cycles, valerate estrogen was administered orally until the endometrial thickness reached up to $7 \mathrm{~mm}$, dydrogesterone was administered orally, together with progesterone was administered vaginally for luteal phase support. For women underwent OS protocols, human menopausal gonadotropin was started on day 3-5, dose of HMG was adjusted according to the follicles diameters and the levels of serum hormone steroids. Urinary hCG was administrated at when follicles diameters reached at least $18 \mathrm{~mm}$.

Serum hormone levels were assessed 3-5 days before embryo transfer, including E2, progesterone (P4) and luteinizing hormone (LH), in the hospital clinical chemistry laboratory. Endometrial thickness was also measured before FET by highly trained sonographers via transvaginal ultrasound (Acuson X300, Siemens, Germany). In our hospital, all embryo transfer procedures were performed under transabdominal ultrasound guidance, despite transvaginal ultrasound guidance can improve the percentage of pregnancies per transfer $[13,14]$.

\section{Data collection and variable definition}

All participants were interviewed in person to obtain information on sociodemographic characteristics (including 
maternal age at oocyte retrieval and embryo transfer, residence, educational level, occupation, and smoking status during pregnancy), reproductive history (including parity, previous abortions, previous ectopic pregnancy, cause of infertility, duration of infertility, and primary infertility). Before initiating IVF cycles, height and weight were measured for each patient, and the patient's body mass index (BMI) was then calculated.

Details on the use of oocyte retrieval and frozenthawed embryo transfer were abstracted from the patient's hospital records as we previously described [15]. ART procedures were conducted using routine protocols, and information was documented, including the $\mathrm{COH}$ protocol, type of insemination, number of oocytes retrieved, the day of embryo transfer, and the number of embryos transferred.

Pregnancy-related complications were abstracted from the participants' health records, including gestational hypertensive disorder, gestational diabetes mellitus (GDM), intrahepatic cholestasis of pregnancy (ICP), meconium staining of the amniotic fluid, preterm birth, and mode of delivery. The birth weight and sex of all neonates were also recorded. The weight for gestational age of each neonate was defined according to a global reference for birth weight for a given gestational age and $\operatorname{sex}[16]$.

All information on the sociodemographic characteristics, reproductive history, ARTs procedures, and the diagnosis of pregnancy-related complications, were exported from the electronic database by department of information.

\section{Statistical analysis}

Continuous variables with normal distributions are represented as the means \pm standard deviations, and differences were tested by one-way analysis of variance. Continuous variables with skewed distributions are shown as medians with interquartile ranges, and differences were tested by the Kruskal-Wallis test. Categorical outcome variables are represented as frequencies with proportions, and the Cochran-Mantel-Haenszel $\mathrm{X}^{2}$ test was used to detect the differences. Multiple comparisons of pregnancy outcomes among groups were detected by multinomial logistic regression.

The outcomes of pregnancy-related complications as well as neonatal birth weight were assessed according to the stratification of singleton or multiple deliveries. To explore the associations between the different endometrial preparation protocols and adverse pregnancy-related complications for both singletons and multiple deliveries, odds ratios (ORs) and 95\% confidence intervals (CIs) were calculated and adjusted for potential confounding factors for each outcome using multivariable logistic regression. The analysis of neonatal birth weight of singletons was performed by multinomial logistic regression analyses. The analysis of neonatal birth weight of multiples was performed via multilevel logistic regression according to Carlin et al. [17].

All statistical analyses were conducted using SAS software version 9.3 (SAS Institute, Inc., Cary, NC). $P$ values less than 0.05 were considered statistically significant. For multiple comparisons, $P$ values less than 0.017 were considered significantly different.

\section{Results}

The flow of the study participants is shown in Fig. 1. A total of 12,950 FET cycles met the inclusion criteria of this study, and 4732 women with live birth deliveries (2997 singletons and 1735 multiples) were included in the analysis. In all, 207 participants were lost to follow-up.

The pregnancy outcomes per transfer cycle of the three groups are shown in Fig. 2. There were no significant differences in the chemical pregnancy rate ( $\mathrm{NC}$ vs. OS vs. HRT: $42.64 \%$ vs. $44.01 \%$ vs. $43.56 \%$ ), clinical pregnancy rate (NC vs. OS vs. HRT: $37.83 \%$ vs. $38.76 \%$ vs. $38.18 \%$ ) or ongoing pregnancy rate (NC vs. OS vs. HRT: $33.69 \%$ vs. $32.39 \%$ vs. $31.63 \%$ ) among the three groups. However, the rate of live birth was lower in patients who underwent HRT cycles than in those who underwent an NC cycle (HRT vs. NC: $28.15 \%$ vs. $31.16 \%, p<0.001$ ), while a comparable live birth rate was observed between patients who underwent endometrium preparation with the NC or OS protocol (NC vs. OS: $31.16 \%$ vs. $31.01 \%$, $p=0.920$ ). Additionally, patients from not only the OS group (OS vs. NC: $16.44 \%$ vs. $10.89 \%, p=0.001$ ) but also the HRT group (HRT vs. NC: 17.14 vs. $10.89 \%, p<$ 0.001 ) had a higher early pregnancy loss rate than was observed in the NC group. The rate of ectopic pregnancy was similar among the three groups.

To analyze the associations among different endometrial preparation protocols in FET cycles and adverse pregnancy-related complications as well as perinatal outcomes, 4732 women with live birth deliveries were included in the analysis. Table 1 shows the distributions of maternal sociodemographic characteristics and reproductive histories among the groups. Notably, differences in maternal age at oocyte retrieval and embryo transfer were small and unlikely to be clinically significant. The causes of infertility were distributed differently among the groups. Anovulatory patients were more likely to undergo endometrium preparation with an OS or HRT protocol than the $\mathrm{NC}$ protocol (NC vs. OS vs. HRT: $1.25 \%$ vs. $8.33 \%$ vs. $11.36 \%, p<0.001)$. However, patients with normal ovarian function, such as tubal infertility or male-factor infertility, were more likely to undergo endometrium preparation with the $\mathrm{NC}$ protocol $(p<$ $0.001)$. 


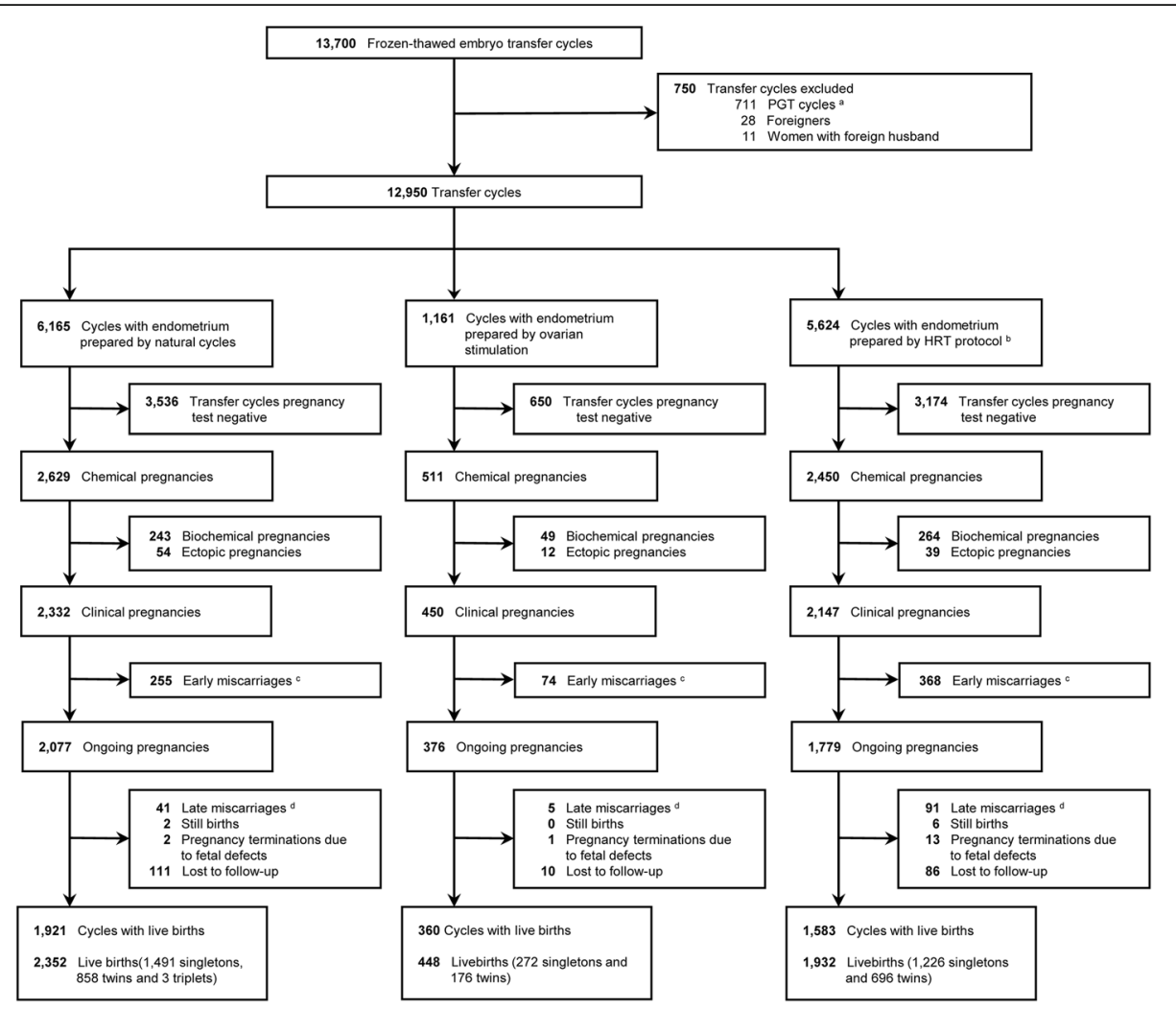

Fig. 1 Study flow chart. a PGT, preimplantation genetic testing. $\mathbf{b}$ HRT, hormone replacement therapy. $\mathbf{c}$ Early miscarriage was defined as spontaneous loss of pregnancy before 12 gestational weeks. $\mathbf{d}$ Late miscarriage was defined as pregnancy loss between 12 and 28 gestational weeks

Table 2 presents the distributions of the procedures used for oocyte retrieval and frozen-thawed embryo transfer among each group. The distribution of $\mathrm{COH}$ protocols $(p=0.345)$, type of insemination $(p=0.901)$ and number of oocytes retrieved $(p=0.171)$ were similar among the groups. With regard to FET procedures, no difference was found in the day of embryo transfer. $(p=0.724)$, number of embryo transferred $(p=0.811)$ or thickness of the endometrium $(p=0.952)$ among the three groups. However, the hormone levels obtained before FET were significantly different among the endometrial preparation protocols. The results showed that E2 levels were significantly higher in the OS and HRT groups than in the NC group (NC vs. OS vs. HRT: 1.20 [0.86-1.88] vs. 1.64 [1.01-2.69] vs. 1.86 [1.07-3.22] $\left.\times 10^{3} \mathrm{pmol} / \mathrm{L}, p<0.001\right)$. P4 levels were lower in the HRT group than in the OS and NC groups (NC vs. OS vs. HRT: 2.90 [1.80-3.60] vs. 2.50 [1.50-2.60] vs. 1.50 [0.90-2.40] nmol/L, $p<0.001)$. Additionally, LH levels were also different after endometrial preparation among the groups (NC vs. OS vs. HRT: 14.05 [9.20-24.90] vs. 10.60 [6.90-17.40] vs. 12.00 [7.50-20.30] IU/L, $p<0.001)$.

The results of the multivariable analysis of pregnancyrelated complications among all pregnant women is shown in Table 3. For both singleton and multiple deliveries, the risk of preeclampsia was higher when the endometrium was prepared with an OS (singleton delivery: $\mathrm{aOR}=2.24,95 \% \mathrm{CI}$ : 1.48-3.38; multiple delivery: aOR $=2.91,95 \%$ CI: $1.56-5.41$ ) or HRT protocol (singleton delivery: $\mathrm{aOR}=1.88,95 \% \mathrm{CI}$ : 1.43-2.48; multiple delivery: $\mathrm{aOR}=2.43,95 \% \mathrm{CI}$ : $1.56-3.78$ ) than when an NC protocol was used. Despite the null effect found between different endometrial preparation protocols and the risk of gestational hypertension among singleton deliveries, HRT cycles were found to increase the risk of gestational hypertension among multiple deliveries $(\mathrm{aOR}=1.99$, 95\%CI: 1.19-3.32). Furthermore, preparing the endometrium with an OS or HRT protocol also increased the risk of ICP (OS protocol: singleton delivery: $\mathrm{aOR}=2.09,95 \% \mathrm{CI}: 1.21-$ 3.59, multiple delivery: $\mathrm{aOR}=2.62,95 \% \mathrm{CI}$ : $1.07-6.38$; HRT protocol: singleton delivery: $\mathrm{aOR}=1.85,95 \% \mathrm{CI}$ : $1.29-2.63$, multiple delivery: $\mathrm{aOR}=2.37,95 \% \mathrm{CI}: 1.25-4.48$ ). A positive association was found between meconium staining of the amniotic fluid and the use of HRT cycles in singleton deliveries $(\mathrm{aOR}=1.31,95 \% \mathrm{CI}: 1.06-1.61)$, while no such association was observed for multiple deliveries $(\mathrm{aOR}=1.07,95 \% \mathrm{CI}$ : $0.70-1.63)$. Additionally, we did not find any associations between the different endometrial preparation protocols and 


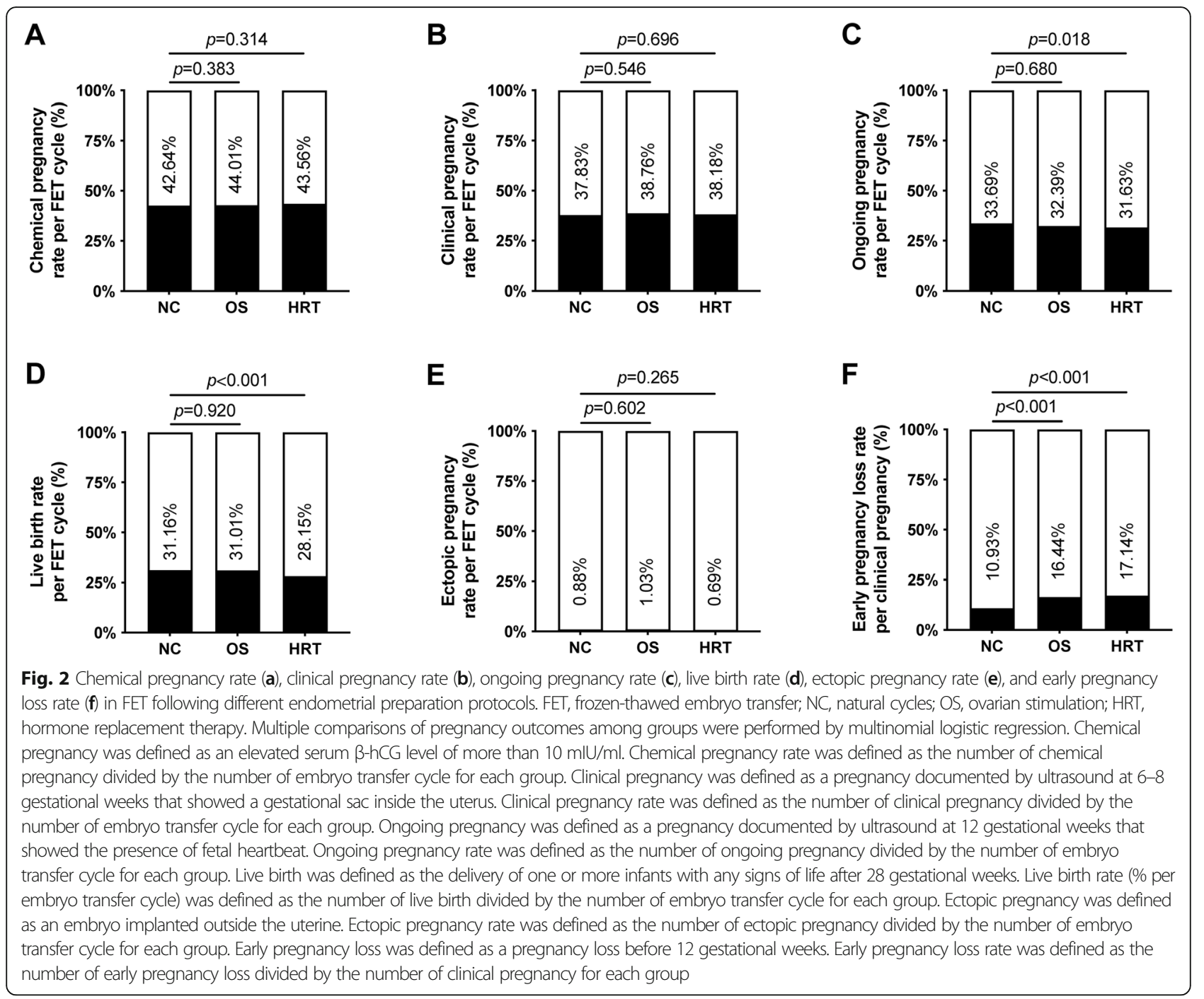

other pregnancy-related complications, including GDM, preterm birth, and cesarean section, for either singleton or multiple deliveries.

Table 4 indicates the associations between different endometrial preparation protocols and neonatal birth weight. Preparing the endometrium with different protocols resulted in similar gender proportions in neonates in both singletons and multiples. The risk of LBW was significantly higher for the OS protocol in both singleton and multiple deliveries (singletons: $\mathrm{aOR}=1.71,95 \% \mathrm{CI}$ : $1.02-$ 2.87; multiples: $\mathrm{aOR}=1.43,95 \% \mathrm{CI}: 1.03-1.99$ ), and the same effect was observed for HRT protocols (singletons: $\mathrm{aOR}=1.93,95 \% \mathrm{CI}: 1.39-2.68$; multiples: $\mathrm{aOR}=1.69$, 95\%CI: 1.37-2.08). Furthermore, preparing the endometrium with the HRT protocol increased the risk of SGA in both singletons (aOR $=1.27,95 \% \mathrm{CI}: 1.05-1.70)$ and multiples $(\mathrm{aOR}=2.33,95 \% \mathrm{CI}: 1.79-3.03)$, and the OS protocol increased the risk of $\mathrm{SGA}$ in singletons $(\mathrm{aOR}=1.58$,
95\%CI: $1.02-2.43)$, but not multiples $(\mathrm{aOR}=1.39,95 \% \mathrm{CI}$ : 0.91-2.13). Additionally, there was no evidence of an association between LGA or macrosomia and endometrial preparation protocols in either singletons or multiples.

We further restricted the analysis to singleton cases and stratified the analysis of pregnancy-related complications (Table S1) and neonatal birth weight (Table S2) according to the number of embryo transfers. In women with single embryo transfer, the risk of preeclampsia, ICP, LBW and SGA were partially eliminated when the endometrium was prepared with an OS or HRT protocol. However, pregnant women who underwent double embryo transfer were still at risk of preeclampsia, ICP, LBW and SGA after an OS or HRT protocol. Additionally, these findings were also confirmed by interactive models that number of FET have interactive effects with OS or HRT protocol on increased risk of preeclampsia, ICP, and LBW (Table S3-4). 
Table 1 Maternal characteristics of pregnancies carried to delivery following FET with different endometrial preparation protocols

\begin{tabular}{|c|c|c|c|c|}
\hline & NC $(N=1921)$ & OS $(N=360)$ & HRT $(N=1583)$ & $p$ \\
\hline & No. (\%) & No. (\%) & No. (\%) & \\
\hline \multicolumn{5}{|l|}{ Maternal socio-demographic characteristics } \\
\hline Age of oocyte retrieval, Mean $\pm S D$, years & $31.25 \pm 3.74$ & $30.95 \pm 3.81$ & $30.73 \pm 3.69$ & $<0.001$ \\
\hline Age of embryo transfer, Mean $\pm S D$, years & $31.43 \pm 3.74$ & $31.04 \pm 3.78$ & $30.96 \pm 3.70$ & $<0.001$ \\
\hline Pre-gestational BMI, Mean $\pm \mathrm{SD}, \mathrm{kg} / \mathrm{m}^{2}$ & $22.42 \pm 3.02$ & $22.55 \pm 2.99$ & $22.35 \pm 3.24$ & 0.216 \\
\hline \multicolumn{5}{|l|}{ Residence } \\
\hline Residents & $1271(66.16)$ & $228(63.33)$ & $1057(66.77)$ & \multirow[t]{2}{*}{0.731} \\
\hline Immigrants & $650(33.84)$ & $132(36.67)$ & $526(33.23)$ & \\
\hline \multicolumn{5}{|l|}{ Education attainment } \\
\hline Primary school or lower & $21(1.09)$ & $8(2.22)$ & $16(1.01)$ & \multirow[t]{4}{*}{0.386} \\
\hline Middle school & $223(11.61)$ & $43(11.94)$ & $207(13.08)$ & \\
\hline High school & $316(16.45)$ & $57(15.83)$ & $271(17.12)$ & \\
\hline Collage or above & $1361(70.85)$ & $252(70.00)$ & $1089(69.79)$ & \\
\hline \multicolumn{5}{|l|}{ Occupation } \\
\hline Employed & $1339(69.70)$ & 219 (60.83) & $1127(71.19)$ & \multirow[t]{3}{*}{0.735} \\
\hline Self-employed & $400(20.82)$ & $108(30.00)$ & $297(18.76)$ & \\
\hline Unemployed & $182(9.47)$ & $33(9.17)$ & $159(10.04)$ & \\
\hline \multicolumn{5}{|l|}{ Smoking during pregnancy } \\
\hline No & $1898(98.80)$ & $357(99.17)$ & $1571(99.24)$ & \multirow[t]{2}{*}{0.187} \\
\hline Yes & $23(1.20)$ & $3(0.83)$ & $12(0.76)$ & \\
\hline \multicolumn{5}{|l|}{ History of reproduction } \\
\hline \multicolumn{5}{|l|}{ Parity } \\
\hline No & $1754(91.83)$ & $333(92.50)$ & $1475(93.18)$ & \multirow[t]{2}{*}{0.132} \\
\hline Yes & $157(8.17)$ & $27(7.50)$ & $108(6.82)$ & \\
\hline \multicolumn{5}{|l|}{ Number of previous abortions } \\
\hline 0 & $1298(67.57)$ & $227(63.06)$ & $1093(69.05)$ & \multirow[t]{3}{*}{0.147} \\
\hline $1-2$ & $560(29.15)$ & $124(34.44)$ & $448(28.30)$ & \\
\hline$\geq 3$ & $63(3.28)$ & $9(2.50)$ & $42(2.65)$ & \\
\hline \multicolumn{5}{|l|}{ Previous ectopic pregnancy } \\
\hline No & $1668(86.83)$ & $316(87.78)$ & $1378(87.05)$ & \multirow[t]{2}{*}{0.837} \\
\hline Yes & $253(13.17)$ & $44(12.22)$ & $205(12.95)$ & \\
\hline \multicolumn{5}{|l|}{ Duration of infertility } \\
\hline $1-2$ & $747(38.89)$ & $133(36.94)$ & $589(37.21)$ & \multirow[t]{3}{*}{0.195} \\
\hline $3-4$ & $592(30.82)$ & $133(36.94)$ & $532(33.61)$ & \\
\hline$\geq 5$ & $582(30.30)$ & $94(26.11)$ & $462(29.19)$ & \\
\hline \multicolumn{5}{|l|}{ Primary infertility } \\
\hline No & $1104(57.47)$ & $207(57.50)$ & $924(58.37)$ & \multirow[t]{2}{*}{0.954} \\
\hline Yes & $817(42.53)$ & $153(42.50)$ & $659(41.63)$ & \\
\hline \multicolumn{5}{|l|}{ Causes of infertility } \\
\hline Tubal infertility & $924(48.10)$ & $155(43.06)$ & $654(41.31)$ & \multirow[t]{6}{*}{$<0.001$} \\
\hline Anovulatory & $24(1.25)$ & $30(8.33)$ & $183(11.56)$ & \\
\hline Endometriosis & $55(2.86)$ & $4(1.11)$ & $44(2.78)$ & \\
\hline Male-factor infertility & $159(8.28)$ & $19(5.28)$ & $106(6.70)$ & \\
\hline Unexplained infertility & $321(16.71)$ & $70(19.44)$ & 196 (12.38) & \\
\hline Combined $^{\mathrm{a}}$ & $438(22.80)$ & 82 (22.78) & $400(25.27)$ & \\
\hline
\end{tabular}

FET frozen embryo transfer, NC natural cycles, OS ovarian stimulation, HRT hormonal replacement therapy, BMI body mass index ${ }^{a}$ Combined was defined as two or more infertile causes mentioned above 
Table 2 ART procedures of pregnancies carried to delivery following FET with different endometrial preparation protocols

\begin{tabular}{|c|c|c|c|c|}
\hline & $N C(N=1921)$ & OS $(N=360)$ & HRT $(N=1583)$ & $p$ \\
\hline & No. (\%) & No. (\%) & No. (\%) & \\
\hline \multicolumn{5}{|l|}{ Characteristics of oocytes retrieval cycle } \\
\hline \multicolumn{5}{|l|}{$\mathrm{COH}$ protocol } \\
\hline GnRH-agonist regimen & $897(46.69)$ & $181(50.28)$ & $743(46.94)$ & \multirow[t]{4}{*}{0.345} \\
\hline GnRH-antagonist regimen & $904(47.06)$ & $166(46.11)$ & $732(46.24)$ & \\
\hline Microflare protocol & $100(5.21)$ & $11(3.06)$ & $85(5.37)$ & \\
\hline Others & $20(1.04)$ & $2(0.56)$ & $23(1.45)$ & \\
\hline \multicolumn{5}{|l|}{ Type of insemination } \\
\hline IVF & $1276(66.42)$ & $240(66.67)$ & $1063(67.15)$ & \multirow[t]{2}{*}{0.901} \\
\hline ICSI & $645(33.58)$ & $120(33.33)$ & $520(32.85)$ & \\
\hline \multicolumn{5}{|l|}{ Number of oocytes retrieved } \\
\hline$\leq 10$ & $712(37.06)$ & $123(34.17)$ & $535(33.80)$ & \multirow[t]{3}{*}{0.171} \\
\hline $11-20$ & $873(45.45)$ & $161(44.72)$ & 739 (46.68) & \\
\hline$>20$ & $336(17.49)$ & $76(21.11)$ & 309 (19.52) & \\
\hline \multicolumn{5}{|l|}{ Characteristics of FET cycle } \\
\hline \multicolumn{5}{|l|}{ Day of embryo transfer } \\
\hline Day3 & $1049(73.35)$ & $258(71.67)$ & $1140(72.02)$ & \multirow[t]{3}{*}{0.724} \\
\hline Day4 & 299 (15.56) & $56(15.56)$ & $267(16.87)$ & \\
\hline Day5 & $213(11.09)$ & $46(12.78)$ & $176(11.12)$ & \\
\hline \multicolumn{5}{|l|}{ Number of embryo transferred } \\
\hline Single embryo transfer & $214(11.14)$ & $33(9.17)$ & $181(11.43)$ & \multirow[t]{2}{*}{0.811} \\
\hline Multiple embryo transfer & $1707(88.86)$ & $327(90.83)$ & $1402(88.57)$ & \\
\hline \multicolumn{5}{|l|}{ Hormone level before embryo transfer } \\
\hline E2, Median $[\mathrm{IQR}], \times 10^{3} \mathrm{pmol} / \mathrm{L}$ & $1.20[0.86-1.88]$ & $1.64[1.01-2.69]$ & $1.86[1.07-3.22]$ & $<0.001$ \\
\hline P4, Median [IQR], nmol/L & $2.90[1.80-3.60]$ & $2.50[1.50-2.60]$ & $1.50[0.90-2.40]$ & $<0.001$ \\
\hline LH, Median [IQR], IU/L & $14.05[9.20-24.90]$ & $10.60[6.90-17.40]$ & $12.00[7.50-20.30]$ & $<0.001$ \\
\hline Endometrial thickness, Mean $\pm \mathrm{SD}, \mathrm{mm}$ & $9.54 \pm 1.46$ & $9.52 \pm 1.52$ & $9.55 \pm 1.43$ & 0.952 \\
\hline
\end{tabular}

FET frozen embryo transfer, NC natural cycles, OS ovarian stimulation, HRT hormonal replacement therapy, $C O H$ controlled ovarian stimulation, IVF in vitro fertilization, ICSI intracytoplasmic sperm injection, E2 estradiol, P4 progesterone, LH luteinizing hormone

\section{Discussion}

In this retrospective cohort of 12,950 FET cycles, we found that the live birth rate after FET was slightly higher and the early pregnancy loss rate significantly lower in patients in whom the endometrium was prepared with an NC protocol than when HRT or OS protocols were used. In addition, among patients with live births, compared to HRT or OS protocols, preparing the endometrium with the $\mathrm{NC}$ protocol were found to be associated with the decreased risk of pregnancy-related complications, including gestational hypertension, preeclampsia, and ICP, as well as the decreased risk of LBW and SGA.

The approach used to prepare the endometrium for FET is often personalized for patients. Commonly, natural cycles are only applied to ovulatory women with regular menstruation, while HRT protocols are performed in all women regardless of menstrual regularity and offers flexibility for FET [5]. Another endometrial preparation protocol of ovarian stimulation is also used to improve certain defects in the follicular and subsequent luteal phase, thus enhancing endometrial receptivity [18]. This is the reason for our findings indicating a higher proportion of women with anovulatory in OS or HRT cycle groups than in the NC groups. Furthermore, the most critical discrepancy among different endometrial preparation protocols is the alteration of maternal hormone levels. Unlike natural cycles, in which the endocrine preparation of the endometrium is achieved by endogenous hormones from a developing follicle, in HRT protocols, the endometrium is prepared with exogenous estradiol and progesterone, while in OS protocols, the follicle cohort is induced with exogenous gonadotropins or an aromatase inhibitor [18, 19]. It has been demonstrated that both estradiol and progesterone levels are aberrant in either HRT or OS protocols $[9,11]$. Although FET is thought to prevent patients 


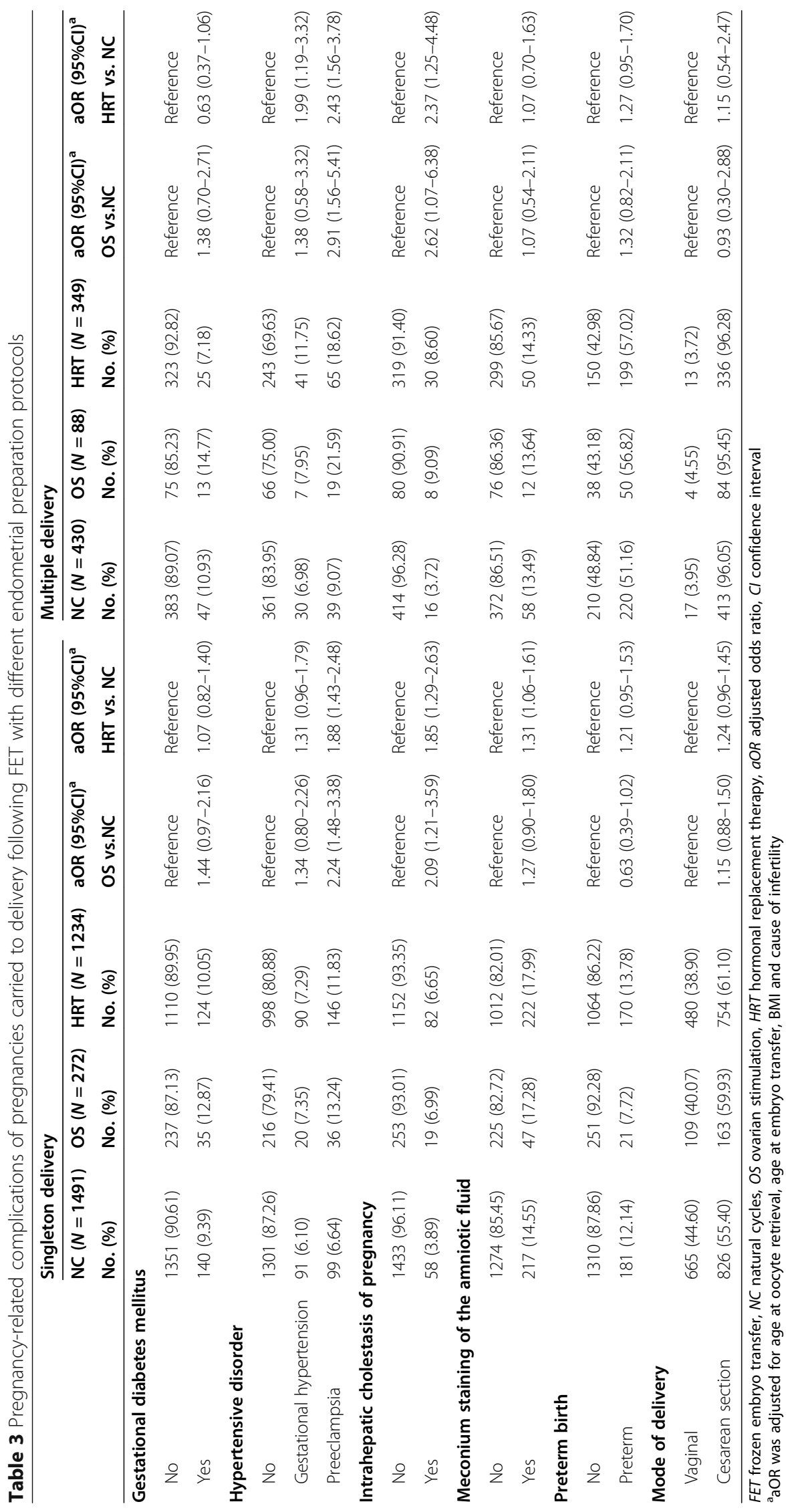




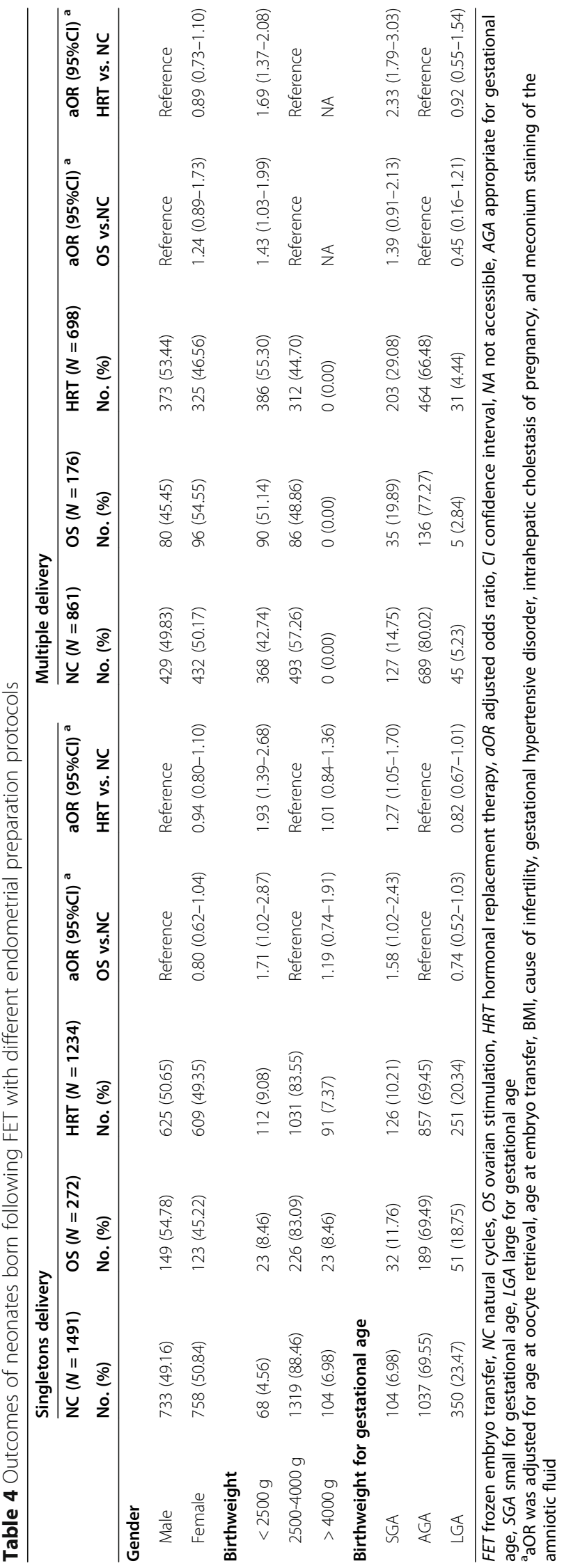


from experiencing the superphysiological hormonal milieu observed in fresh embryo transfer, preparing the endometrium with HRT or OS protocols still leads to alterations in maternal hormone levels. Thus, in our study, serum estradiol levels were elevated after the endometrium was prepared with HRT or OS protocols. The levels of LH and progesterone were significantly lower in the HRT group than in NC group due to the suppression of normal regulation.

Different endometrial preparation regimens are used to obtain optimal conditions for embryo implantation following FET. However, there is no conclusive evidence indicating that one of these approaches is superior to another. A meta-analysis including 7 retrospective studies and 1 randomized controlled trial revealed that comparable results with regard to the clinical pregnancy rate, ongoing pregnancy rate, and livebirth rate were achieved between NC and HRT protocols [20]. Additionally, another metaanalysis failed to find any superiority between the effects of NC and OS protocols on the pregnancy rate [5]. Based on our findings, although these three protocols produced similar rates of chemical pregnancy, clinical pregnancy, and ongoing pregnancy, we found that the HRT protocol achieved a lower rate of live birth than was obtained using the NC and OS protocols. Furthermore, the chance of early pregnancy loss was also higher for OS and HRT protocols. Ezoe et al. and Horcajadas et al. reported that decidualization might be impaired, leading to a subsequent decrease in endometrial receptivity after ovarian stimulation [11, 21]. This might help to explain the increased rate of early pregnancy loss observed after OS protocols. However, a possible reason for the higher rate of early pregnancy loss observed in HRT cycles might be the excessive estradiol in the environment or a suboptimal progesterone to estradiol ratio $[7,22]$.

Compared to the attention around regarding the effects of different endometrial preparation protocols on pregnancy outcomes following FET, pregnancy-related complications are rarely considered. Recently, Saito et al. first reported that preparing the endometrium with HRT was associated with higher risks of hypertensive disorders and placenta accrete and a lower risk of GDM [23]. In contrast to Saito et al., who excluded the OS protocol, our study included all FET cycles with OS regimens despite their limited number. Our study adds some interesting new findings to the literature following on Saito et al., in which these cycles were not mentioned. In addition to hypertensive disorders, including gestational hypertension and preeclampsia, we also found that the risk of ICP was higher when using both OS and HRT protocols than when using the NC protocol. However, we did not find any association between GDM and OS or HRT protocols. Our previous study indicated that $\mathrm{COH}$-induced high estradiol levels are sustained for more than 8 weeks following fresh embryo transfer and can lead to adverse effects on endometrial receptivity and intrauterine fetal growth [24]. Although FET could effectively protect patients from the adverse effects of high maternal E2 during early pregnancy, estrogen supplementation during the period of HRT and OS-induced higher estradiol levels would still affect maternal serum estradiol levels to some extent. In vivo studies performed in nonhuman primates have indicated that activated E2 signaling might be involved in regulating trophoblast differentiation in early pregnancy and subsequently results in the insufficient invasion of the trophoblast and uteroplacental vessel remodeling $[25,26]$. Impaired uteroplacental blood flow might influence intrauterine fetal growth and several obstetric outcomes, including preeclampsia and SGA [27]. Thus, it is not surprising to find that the risk of LBW and SGA was higher following the OS and HRT protocols in our study. Importantly, multiple pregnancy is one of the critical risk factors for maternal and fetal comorbidities including gestational hypertensive disorders, LBW and SGA $[28,29]$, thus that is why the incidence of gestational hypertensive disorders, LBW and SGA reported higher in multiple deliveries stratification in our study. In addition, elevated estradiol level was also regarded to be negatively associated with bile salt export pump, which is responsible for biliary secretion of bile acids, thus subsequently suppressed the enterohepatic circulation of bile acids and contributed to the ICP [30].

Although preparing the endometrium with $\mathrm{NC}$ provides patients with many benefits with regard to perinatal health, the OS and HRT protocol still have advantages in terms of minimal monitoring and easy scheduling with FET procedures and are especially helpful to patients with irregular menstrual cycles or who are anovulatory. If an OS or HRT protocol is required in the process of FET, we will recommend a single embryo transfer rather than double embryo transfer according to our findings shown in Tables S1 and S2. Single embryo transfer could partially minimize the risk of adverse effects on pregnancy-related complications and neonatal birth weight induced by OS and HRT protocol. A possible explanation of these findings might be the increased chance that embryos will implant on unfavorable sites during multiple embryo transfer than during single embryo transfer. Animal experiments have indicated that embryos implanted at unfavorable sites are associated with pregnancy complications [30].

As this is a hospital-based retrospective cohort study, we failed to rule out some unknown confounders that might influence the risk of obstetric complications and newborn birth weight; these included nutrient intake and physical activities performed during pregnancy. For instance, vitamin D was believed to improve the pregnancy outcomes after embryo transfer [31,32], and some of 
patients administrates vitamin $\mathrm{D}$ to modulate reproductive outcomes. However, as vitamin D could be available overthe-counter, we failed to record the details of vitamin D supplementation, which might cause bias in this analysis. Limited sample size is another limitation in this study, especially for multiple pregnancy stratification, it reduced statistical power when analyzing maternal and neonatal outcomes. Furthermore, bias induced by patients' favoring of different endometrial preparation protocols is another inevitable limitation due to the retrospective design of this study. In addition, hormone levels were measured before FET at a time equivalent to the day of oocyte retrieval, when progesterone supplementation was not yet started. Thus, these findings may not reflect the actual level of progesterone after HRT, and we failed to confirm any associations between the level of progesterone after HRT and any pregnancy outcomes, pregnancy-related complications and neonatal outcomes. Although our study is not the first to reveal that HRT protocols affect obstetric complications, our findings add new information to what is known about the association between OS protocols and obstetric complications and neonatal birth weight. Moreover, our stratified analysis confirms the benefit of single embryo transfer on OS- or HRT-induced adverse effects with regard to complications and neonatal birth weight. Ideally, future randomized control trials exploring the issue of endometrial preparation protocols should give more attention to pregnancy-related complications as one of the study outcomes.

\section{Conclusion}

In summary, our findings showed that with the exception of the application of OS or HRT protocols in women who are anovulatory, preparing the endometrium with natural cycles should be encouraged to avoid the aberrant hormone levels observed before FET in women with regular menstrual cycles. Although the NC protocol requires more frequent visits to the hospital and endocrine and ultrasonographic monitoring, it also helps women to optimize pregnancy outcomes and improve maternal health during pregnancy. Caution should be warranted when using OS or HRT protocols, due to the associations with gestational hypertension, preeclampsia, and ICP as well as LBW and SGA.

\section{Abbreviations}

ART: Assisted reproductive technology; BMI: Body mass index; Cl: Confidence intervals; $\mathrm{COH}$ : Controlled ovarian hyperstimulation; FET: Frozen-thawed embryo transfer; GDM: Gestational diabetes mellitus; HRT: Hormone replacement therapy; ICP: Intrahepatic cholestasis of pregnancy; ICSI: Intracytoplasmic sperm injection; IVF: In vitro fertilization; LBW: Low birth weight; LGA: Large for gestational age; LH: Luteinizing hormone; NC: Natural cycles; OR: Odds ratios; OS: Ovarian stimulation; PGT: Preimplantation genetic testing; SGA: Small for gestational age

\section{Supplementary Information}

The online version contains supplementary material available at https://doi. org/10.1186/s12884-021-03791-9.

Additional file 1: Table S1. Pregnancy-related complications of singleton deliveries following FET with different endometrial preparation protocols stratified according to the number of embryo transfer. Table S2. Outcomes of singletons born following FET with different endometrial preparation protocols stratified according to the number of embryo transfer. Table S3. Interactive effect of number of FET with different endometrial preparation protocols on pregnancy-related complications of singleton deliveries following FET. Table S4. Interactive effect of number of FET with different endometrial preparation protocols on outcomes of singletons born following FET

\section{Acknowledgements}

Not applicable.

\section{Authors' contributions}

$\mathrm{HFH}$ conceived the hypothesis. YTW designed the study. CL, JJX and YCH drafted the manuscript. YW, HL, CCD and JW conducted the statistical analysis and participated in the discussion. CYS and LC participated in data collection. JZS and HFH critically revised the manuscript for important intellectual content. All authors commented on the drafts and approved the final draft.

\section{Funding}

This study was supported by the National Natural Science Foundation of China $(82001648,81671412,81661128010)$, the Interdisciplinary Key Program of Shanghai Jiao Tong University (YG2014ZD08), and Ministry of Education Key Laboratory of Reproductive Genetics (ZDFY2020-RG-0002). The funding bodies played no role in the design of the study and collection, analysis, and interpretation of data and in writing the manuscript.

\section{Availability of data and materials}

The data can be available from the corresponding author on the resealable request.

\section{Declarations}

Ethics approval and consent to participate

Written informed consent about follow-up until delivery was routinely obtained from all women when they initiated their ART cycles in International Peace Maternity and Child Health Hospital. Follow-up and data collection in this study involving human participants were conducted with the approval of the Institutional Review Board of the International Peace Maternity and Child Health Hospital, Shanghai, China (No. GKLW-2016-21).

\section{Consent for publication}

Not applicable.

\section{Competing interests}

The authors declare that they have no competing interests.

\section{Author details}

${ }^{1}$ International Peace Maternity and Child Health Hospital, School of Medicine, Shanghai Jiao Tong University, No.910, Hengshan Rd., Shanghai 200030, China. ${ }^{2}$ Shanghai Key Laboratory of Embryo Original Diseases, Shanghai 200030, China. ${ }^{3}$ Department of Pathology and Pathophysiology, School of Medicine, Zhejiang University, Hangzhou, Zhejiang, China. ${ }^{4}$ Obstetrics and Gynecology Hospital, Fudan University, No.419, Fangxie Rd., Shanghai 200000, China.

Received: 11 June 2020 Accepted: 12 April 2021

Published online: 29 April 2021

References

1. Thurin A, Hausken J, Hillensjo T, et al. Elective single-embryo transfer versus double-embryo transfer in in vitro fertilization. N Engl J Med. 2004:351(23): 2392-402. https://doi.org/10.1056/NEJMoa041032. 
2. Tiitinen A, Halttunen $M$, Harkki P, Vuoristo P, Hyden-Granskog C. Elective single embryo transfer: the value of cryopreservation. Hum Reprod. 2001; 16(6):1140-4. https://doi.org/10.1093/humrep/16.6.1140.

3. Shapiro BS, Daneshmand ST, Garner FC, Aguirre M, Hudson C. Clinical rationale for cryopreservation of entire embryo cohorts in lieu of fresh transfer. Fertil Steril. 2014;102(1):3-9. https://doi.org/10.1016/j.fertnstert.2014. 04.018.

4. Mackens S, Santos-Ribeiro S, van de Vijver A, Racca A, van Landuyt L, Tournaye $\mathrm{H}$, et al. Frozen embryo transfer: a review on the optimal endometrial preparation and timing. Hum Reprod. 2017;32(11):2234-42. https://doi.org/10.1093/humrep/dex285.

5. Yarali H, Polat M, Mumusoglu S, Yarali I, Bozdag G. Preparation of endometrium for frozen embryo replacement cycles: a systematic review and meta-analysis. J Assist Reprod Genet. 2016;33(10):1287-304. https://doi. org/10.1007/s10815-016-0787-0.

6. Mounce G, McVeigh E, Turner K, Child TJ. Randomized, controlled pilot trial of natural versus hormone replacement therapy cycles in frozen embryo replacement in vitro fertilization. Fertil Steril. 2015;104(4):915-20. https://doi. org/10.1016/j.fertnstert.2015.07.1131

7. Morozov V, Ruman J, Kenigsberg D, Moodie G, Brenner S. Natural cycle cryo-thaw transfer may improve pregnancy outcome. J Assist Reprod Genet. 2007;24(4):119-23. https://doi.org/10.1007/s10815-006-9100-y.

8. Hill MJ, Miller KA, Frattarelli JL. A GnRH agonist and exogenous hormone stimulation protocol has a higher live-birth rate than a natural endogenous hormone protocol for frozen-thawed blastocyst-stage embryo transfer cycles: an analysis of 1391 cycles. Fertil Steril. 2010;93(2):416-22. https://doi. org/10.1016/j.fertnstert.2008.11.027.

9. Levron J, Yerushalmi GM, Brengauz M, Gat I, Katorza E. Comparison between two protocols for thawed embryo transfer: natural cycle versus exogenous hormone replacement. Gynecol Endocrinol. 2014;30(7):494-7. https://doi. org/10.3109/09513590.2014.900032.

10. Altmae S, Tamm-Rosenstein K, Esteban FJ, et al. Endometrial transcriptome analysis indicates superiority of natural over artificial cycles in recurrent implantation failure patients undergoing frozen embryo transfer. Reprod BioMed Online. 2016;32(6):597-613. https://doi.org/10.1016/j.rbmo.2016.03.004.

11. Ezoe K, Daikoku T, Yabuuchi A, Murata N, Kawano H, Abe T, et al. Ovarian stimulation using human chorionic gonadotrophin impairs blastocyst implantation and decidualization by altering ovarian hormone levels and downstream signaling in mice. Mol Hum Reprod. 2014;20(11):1101-16. https://doi.org/10.1093/molehr/gau065.

12. Duan CC, Li C, He YC, et al. Oocyte exposure to supraphysiological estradiol during ovarian stimulation increased the risk of adverse perinatal outcomes after frozen-thawed embryo transfer: a retrospective cohort study. J Dev Orig Health Dis. 2019:1-11. https://doi.org/10.1017/S2040174419000679.

13. Cozzolino M, Vitagliano A, Di Giovanni MV, et al. Ultrasound-guided embryo transfer: summary of the evidence and new perspectives. A systematic review and meta-analysis. Reprod BioMed Online. 2018;36(5):524-42. https:// doi.org/10.1016/j.rbmo.2018.01.015.

14. Larue L, Keromnes G, Massari A, Roche C, Moulin J, Gronier H, et al. Transvaginal ultrasound-guided embryo transfer in IVF. J Gynecol Obstet Hum Reprod. 2017;46(5):411-6. https://doi.org/10.1016/j.jogoh.2017.02.015.

15. Wu YT, Li C, Zhu YM, Zou SH, Wu QF, Wang LP, et al. Outcomes of neonates born following transfers of frozen-thawed cleavage-stage embryos with blastomere loss: a prospective, multicenter, cohort study. BMC Med. 2018;16(1):96. https://doi.org/10.1186/s12916-018-1077-8.

16. Mikolajczyk RT, Zhang J, Betran AP, Souza JP, Mori R, Gülmezoglu AM, et al. A global reference for fetal-weight and birthweight percentiles. Lancet. 2011;377(9780):1855-61. https://doi.org/10.1016/S0140-6736(11)60364-4.

17. Carlin JB, Gurrin LC, Sterne JA, Morley R, Dwyer T. Regression models for twin studies: a critical review. Int J Epidemiol. 2005;34(5):1089-99. https:// doi.org/10.1093/ije/dyi153.

18. Van der Auwera I, Meuleman C, Koninckx PR. Human menopausal gonadotrophin increases pregnancy rate in comparison with clomiphene citrate during replacement cycles of frozen/thawed pronucleate ova. Hum Reprod. 1994;9(8):1556-60. https://doi.org/10.1093/oxfordjournals. humrep.a138748.

19. Younis JS, Simon A, Laufer N. Endometrial preparation: lessons from oocyte donation. Fertil Steril. 1996;66(6):873-84. https://doi.org/10.1016/s0015-0282 (16)58677-4

20. Groenewoud ER, Cantineau AE, Kollen BJ, Macklon NS, Cohlen BJ. What is the optimal means of preparing the endometrium in frozen-thawed embryo transfer cycles? A systematic review and meta-analysis. Hum Reprod Update. 2013;19(5):458-70. https://doi.org/10.1093/humupd/dmt030.

21. Horcajadas JA, Minguez P, Dopazo J, et al. Controlled ovarian stimulation induces a functional genomic delay of the endometrium with potential clinical implications. J Clin Endocrinol Metab. 2008;93(11):4500-10. https:// doi.org/10.1210/jc.2008-0588.

22. Tomas C, Alsbjerg B, Martikainen H, Humaidan P. Pregnancy loss after frozen-embryo transfer--a comparison of three protocols. Fertil Steril. 2012; 98(5):1165-9. https://doi.org/10.1016/j.fertnstert.2012.07.1058.

23. Saito K, Kuwahara A, Ishikawa T, Morisaki N, Miyado M, Miyado K, et al. Endometrial preparation methods for frozen-thawed embryo transfer are associated with altered risks of hypertensive disorders of pregnancy, placenta accreta, and gestational diabetes mellitus. Hum Reprod. 2019;34(8): 1567-75. https://doi.org/10.1093/humrep/dez079.

24. Hu XL, Feng $\mathrm{C}$, Lin XH, Zhong ZX, Zhu YM, Lv PP, et al. High maternal serum estradiol environment in the first trimester is associated with the increased risk of small-for-gestational-age birth. J Clin Endocrinol Metab. 2014;99(6):2217-24. https://doi.org/10.1210/jc.2013-3362.

25. Bonagura TW, Babischkin JS, Aberdeen GW, Pepe GJ, Albrecht ED. Prematurely elevating estradiol in early baboon pregnancy suppresses uterine artery remodeling and expression of extravillous placental vascular endothelial growth factor and alpha1beta1 and alpha5beta1 integrins. Endocrinology. 2012;153(6):2897-906. https://doi.org/10.1210/en.2012-1141.

26. Maliqueo M, Echiburu B, Crisosto N. Sex steroids modulate uterine-placental vasculature: implications for obstetrics and neonatal outcomes. Front Physiol. 2016;7:152.

27. Bonagura TW, Pepe GJ, Enders AC, Albrecht ED. Suppression of extravillous trophoblast vascular endothelial growth factor expression and uterine spiral artery invasion by estrogen during early baboon pregnancy. Endocrinology. 2008;149(10):5078-87. https://doi.org/10.1210/en.2008-0116.

28. Phipps EA, Thadhani R, Benzing T, Karumanchi SA. Pre-eclampsia: pathogenesis, novel diagnostics and therapies. Nat Rev Nephrol. 2019;15(5): 275-89. https://doi.org/10.1038/s41581-019-0119-6.

29. Ooki S. The effect of an increase in the rate of multiple births on low-birthweight and preterm deliveries during 1975-2008. J Epidemiol. 2010;20(6): 480-8. https://doi.org/10.2188/jea.JE20100022.

30. Chen Q, Zhang Y, Peng H, Lei L, Kuang H, Zhang L, et al. Transient \{beta\}2adrenoceptor activation confers pregnancy loss by disrupting embryo spacing at implantation. J Biol Chem. 2011;286(6):4349-56. https://doi.org/1 $0.1074 / j b c . M 110.197202$

31. Lagana AS, Vitale SG, Ban FH, Vrtacnik-Bokal E, D'Anna R. Vitamin D in human reproduction: the more, the better? An evidence-based critical appraisal. Eur Rev Med Pharmacol Sci. 2017;21(18):4243-51.

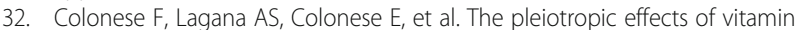
$D$ in gynaecological and obstetric diseases: an overview on a hot topic. Biomed Res Int. 2015;2015:986281.

\section{Publisher's Note}

Springer Nature remains neutral with regard to jurisdictional claims in published maps and institutional affiliations.

Ready to submit your research? Choose BMC and benefit from:

- fast, convenient online submission

- thorough peer review by experienced researchers in your field

- rapid publication on acceptance

- support for research data, including large and complex data types

- gold Open Access which fosters wider collaboration and increased citations

- maximum visibility for your research: over $100 \mathrm{M}$ website views per year

At BMC, research is always in progress.

Learn more biomedcentral.com/submission 